\title{
THE SEARCH FOR EXOPLANETARY RADIO EMISSIONS
}

\author{
P. Zarka*
}

\begin{abstract}
A chronological review of this relatively recent subject is presented, from the discovery of the first exoplanet - orbiting a pulsar - to theoretical predictions and ongoing searches conducted with most large low-frequency radiotelescopes. The specific interest of radio observations is emphasized.
\end{abstract}

\section{Historical Landmarks}

"Pre"-history of the field started with Yantis et al. [1977], Winglee et al. [1986], Lecacheux [1991], and Burke [1992], all making an analogy between Jupiter and putative exoplanets and suggesting to look for radio emissions of the latter. Most of them noticed that the high intensity of the galactic background is a serious drawback to the detectability of such emissions (see below). Winglee et al. [1986] conducted in addition a first search using the VLA at $330 \mathrm{MHz}$ and $1.4 \mathrm{GHz}$, with negative results. The first exoplanet was discovered around the pulsar PSR1257+12, by pulse arrival timing, using the Arecibo radiotelescope at $430 \mathrm{MHz}$ [Wolszczan and Frail, 1992]. In the same year, a Nançay-Meudon/Graz/Kharkov collaboration started around the use of the UTR-2 radiotelescope [Braude et al., 1978] together with modern Nançay receivers. The first tentative exoplanet search at UTR-2 started in 1995, in parallel with Jupiter studies, using an Acousto-Optical Spectrograph (AOS - [Raterron, 1985]) in the band 18-28 MHz. Its principles and method were presented at the first French workshop on exoplanet search, held at the Observatoire de Haute-Provence [Zarka, 1995]. In october 1995, the discovery of $51 \mathrm{Peg}$ B around a solar type star [Mayor and Queloz, 1995] marked the true birth of a new field, which counts today >500 known exoplanets (see exoplanet.eu).

The radio search for exoplanets was much developed at UTR-2, using an AOS with a dual beam (simultaneous On \& Off) observation strategy and specific processing algorithms for mitigating the effect of Radio Frequency Interference (RFI) and ionospheric perturbations, and for weak burst detection. The method and preliminary results (negative) were published in [Zarka et al., 1997] together with theoretical considerations about the

* LESIA, Observatoire de Paris, UMR CNRS 8109, 92195 Meudon, France 
detectability of Jupiter-like radio emissions. Due to the strong decameter-wave galactic background (see e.g. [Manning and Dulk, 2001]), Jupiter's decameter radio emission, in spite of its huge intensity (several $10^{6} \mathrm{Jy}$ [Zarka, 1998; Zarka et al, 2004], with $1 \mathrm{Jy}=$ $10^{-26} \mathrm{Wm}^{-2} \mathrm{~Hz}^{-1}$ ), is undetectable beyond $\sim 0.5 \mathrm{pc}$. A central question is thus: "could there be planetary radio emissions much stronger than Jupiter's ?"

\section{Theoretical Predictions}

This question started to be addressed in more details in 1997, in the frame of one of the first working groups of the International Space Science Institute (ISSI: www.issibern.ch) "Theory and Observations of Solar System Radio Emissions", coordinated by H. Rucker. It was soon realized that, in spite of the great effectiveness of the cyclotron maser instability (CMI) as a generation mechanism for magnetospheric/auroral radio emissions, their flux cannot be predicted from first principles and basic planetary parameters. This is due to the strong dependence of the produced radio flux on the detailed shape of unstable electron distributions in source regions and on the small scale source structure [Zarka et al., 1986; Le Quéau, 1988; Hilgers, 1992; Louarn, 1997; Galopeau et al., 2004]. The former is itself strongly dependent on electron acceleration processes (parallel electric fields, magnetic reconnection, magnetospheric compressions...). Instead, scaling laws were established from measured properties of planetary radio emissions in our Solar system and, based on physically sound assumptions, were extrapolated to the case of exoplanets.

The first of these scaling laws was proposed by Desch and Kaiser [1984] for predicting the power of Uranus' radio emissions from extrapolation of Earth's, Jupiter's and Saturn's radio powers in the kilo-hectometer wavelength range. This law related the flux of kinetic energy of the solar wind intercepted by the magnetospheric cross-section $\left(P_{\text {ram }}\right)$ to the median auroral radio power $\left(P_{\text {radio }}\right)$ integrated over its entire bandwidth and emission (beaming) diagram. It was extended by Zarka [1992, 1998] who incorporated Uranus' and Neptune's radio emissions as well as Jupiter's decameter emissions. This led to a scaling law $P_{\text {radio }} \sim \eta \times P_{\text {ram }}^{\alpha}$ ) with slope (in log scale) $\alpha \sim 1$ and a constant of proportionality $\eta \sim 10^{-5}$. The latter authors also noticed that a larger source "homogeneity" - defined as the ratio between the planetary magnetic field gradient length and the radio wavelength favours a larger emitted flux. Zarka et al. [1997] and Farrell et al. [1999] extrapolated this "radio-to-kinetic" scaling law to the newly discovered exoplanets, and found that a radio power $10^{2-3}$ times larger than Jupiter's could be produced under favourable circumstances by an exoplanet in orbit around a solar-type star. Crucial parameters favouring a large radio power are the planet's orbital distance - especially significant with the discovery of "hot jupiters" - and a large planetary magnetic moment (estimated via several variants of the Blackett scaling law to be $\propto \omega^{1 / 2-1} m^{1-2}$, with $\omega$ the planetary rotation's angular frequency and $m$ the planetary mass Farrell et al. [1999]). Zarka et al. [1997] noted that an additional factor $\times 10^{2-3}$ can be gained with a faster/denser stellar wind, while Farrell et al. [1999] noted that interstellar scintillations could lead to temporary flux increases by a factor $\sim \times 10^{2}$.

The reflexion was pursued in the frame of the above ISSI group, and it was found that: (i) auroral/magnetospheric radio powers of solar system planets were also proportional 
to the Poynting flux of the Solar wind on the magnetospheric cros-section; and (ii) the same relation of proportionality also relates satellite-induced (Io, Ganymede) radio emissions in the magnetosphere of Jupiter with the incident Poynting flux convected by the rotation of the planetary magnetic field on the obstacle (ionosphere of Io, magnetosphere of Ganymede). Zarka et al. [2001] thus proposed a general frame for considering plasma flow-obstacle interactions, in which strong radio emission is produced via the CMI if either the flow or the obstacle is strongly magnetized. In this frame, the radio power $\left(P_{r}\right)$ is simply proportional to the Poynting flux onto the obstacle $\left(P_{m}=\pi R_{\text {obstacle }}^{2} V B_{\perp}^{2} / \mu_{\circ}\right)$, with $V$ the solar wind velocity and $B_{\perp}$ the interplanetary magnetic field perpendicular to the flow in the planetary frame (i.e. taking into account the aberration due to the increasing orbital velocity for decreasing orbital distances), and a constant of proportionality $\sim 2-10 \times 10^{-3}$. Considering the evolution with orbital distance of the magnetospheric size, wind velocity and perpendicular magnetic field component, Zarka et al. [2001] and Zarka [2007] found that the solar wind Poynting flux - and, as a consequence of the above "radio-to-magnetic" scaling law, the emitted radio power - of hot jupiters orbiting at 5-10 stellar radii around a solar-type star could be $10^{5}$ times stronger than Jupiter's. Stronger radio emissions may be produced in the case of a faster and/or more strongly magnetized stellar wind.

The detection of such extremely strong emissions against the galactic radio background then becomes possible up to distances of several tens to several hundred parsecs, making it possible to conduct observational searches with large instruments. These searches should be focussed at low frequencies ( $<$ a few tens of $\mathrm{MHz}$ ), as CMI radio emission is restricted to frequencies below the highest cyclotron frequencies available near the planet's surface $\left(f_{c e}=e B / 2 \pi m_{e}\right.$, i.e. $f_{c e}[\mathrm{MHz}]=2.8 B$ [Gauss $\left.]\right)$.

\section{Early Low-frequency Observations}

Imaging observations of several targets (including $\tau$ Boo, 70 Vir, HD 114762, and HD 80606) at the VLA were performed by various teams at 74, 330, and $1465 \mathrm{MHz}$ [Winglee et al., 1986; Bastian et al., 2000; Farrell et al., 2003, 2004a,b; Lazio and Farrell, 2007; Lazio et al., 2010a,b]. No detection was obtained. This can be due to the relatively high observing frequencies and small effective area $\left(\sim 13,000 \mathrm{~m}^{2}\right)$. Sensitivity from $\sim 0.1$ to 1 Jy was achieved.

A large observation campaign, of a dozen exoplanet-hosting stars and a few brown dwarfs, was conducted at UTR-2/Kharkov between 1997 and 2001 [Ryabov et al., 2004]. UTR-2 is a T-shape phased array with $150,000 \mathrm{~m}^{2}$ effective area, operating in the $10-30 \mathrm{MHz}$ range, just above the Earth's ionospheric cutoff (i.e. the lowest frequency range observable from the ground). It has the capability to form several (up to 5) beams in parallel, and thus to point simultaneously at several directions in the sky [Braude et al., 1978]. During this campaign, an AOS was used that could record the output of 2 beams in the 18-28 $\mathrm{MHz}$ band, separated by $1^{\circ}$, allowing us to perform simultaneous On-source \& Off-source observations [Zarka et al., 1997]. Data consisted of "On" and "Off" dynamic spectra with moderate spectral resolution $(\sim 50 \mathrm{kHz})$ and high time resolution (a few msec per 
spectrum). First detection of individual pulsar pulses with the above equipment (Figure 1) proved that sensitivity of a few Jy was achieved with short integrations.

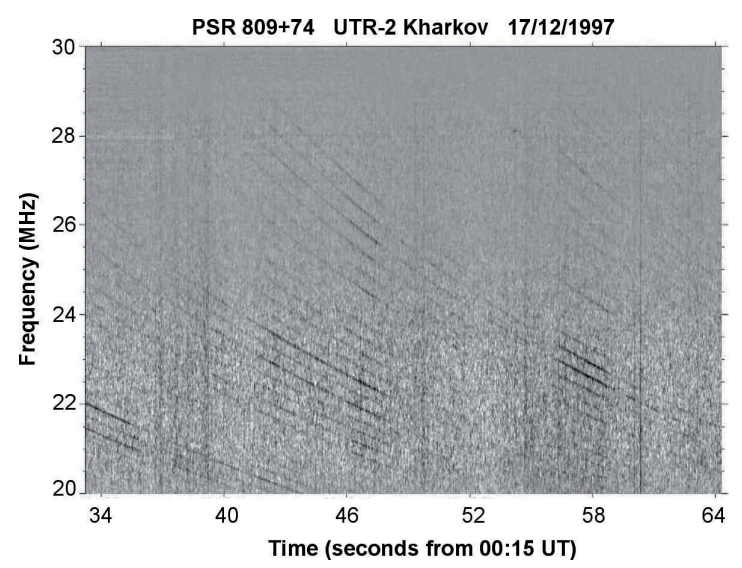

Figure 1: Dispersed individual pulses of pulsar PSR0809+74 detected at UTR-2 in 1997 with an $A O S$.

One key idea of these observations is to observe with an instantaneous bandwidth $b$ and an integration time $\tau$ small enough to (i) perform efficient RFI mitigation in the timefrequency ( $t-f)$ plane (for which specific algorithms were developed), and (ii) ensure that the expected signal is much smaller than the fluctuations of the galactic background $(\propto$ $(b . \tau)^{-1 / 2}$ ) and thus "protected" from the RFI mitigation. As a second step, t-f integration is performed to increase the sensitivity and try to detect weak bursts of emission, for which a statistical approach was developed [Ryabov et al., 2004]. Dedispersion must be applied to $\mathrm{t}-\mathrm{f}$ data at this stage because the expected DM (dispersion measure) for known exoplanets lies in the range 0 to $2 \mathrm{pc} . \mathrm{cm}^{-3}$ (up to $\sim 10$ for the more distant Corot and WASP planets), leading to dispersion delays of 5 to $60 \mathrm{sec}$ between the frequencies 30 $\mathrm{MHz}$ and $20 \mathrm{MHz}$. Temporal broadening of the signals is negligible for the integration times needed here [Zarka et al., 1997]. Comparison of On and Off signals is essential to correct for background fluctuations due to ionospheric propagation (scintillation), as well as for RFI identification and mitigation, and weak burst detection. No convincing detection was obtained in 1997-2001 at UTR-2. We attribute that to the limited time stability of the AOS response ( $\leq$ a few tens of sec), its low spectral resolution $(\sim 50 \mathrm{kHz})$ and a very perturbed ionosphere near Solar maximum.

A new ISSI group was formed in 2004 ("Search for radio emissions from extrasolar planets", led by D. Winterhalter), who performed $153 \mathrm{MHz}$ observations at the GMRT in 2006. A few targets were observed ( $\tau$ Boo, 70 Vir, HD 162020, HD 179949, $v$ And) in imagery and dynamic spectrum. No detection was achieved [Majid et al., 2006; Winterhalter et al., 2006]. George and Stevens [2007, 2008] obtained negative results as well with the same instrument on $\epsilon$ Eri and HD128311. A 10 mJy sensitivity was claimed to be reached with $15 \mathrm{~min}$. integration, but Majid et al. [2006] pointed out the fact that 
$153 \mathrm{MHz}$ data are strongly polluted by RFI. This strong limit to sensitivity as well as the relatively high frequency of observation can explain the absence of any detection.

\section{Tentative Optical Detection and More Theoretical Work}

Shkolnik et al. [2003, 2005] found for 2 solar-type stars (HD 179949 and $v$ And) out of 13 studied a periodic variation of the optical/UV chromospheric activity in Ca lines, at a few \% level. In both cases, the period coincided with the orbital period of the hot jupiter orbiting the star. This result was interpreted as the detection of a chromospheric hot spot at a fixed phase relative to the sub-planet point, and the origin of this hot spot was attributed to star-planet plasma interaction, e.g. via magnetic reconnection between the planetary and stellar fields [Cuntz et al., 2000; Saar and Cuntz, 2001]. The hot spot was found to lie $60^{\circ}$ ahead of the sub-planetary point for HD 179949 , and $170^{\circ}$ ahead for $v$ And, in spite of a planetary orbital period shorter than the stellar rotation. A convincing explanation was provided by Preusse et al. [2006] in terms of the upstream propagation of Alfvén waves excited by the planet in the magnetic field of an expanding stellar wind. Further observations did not confirm this optical signature, casting doubts on the original detection, which was interpreted as an intrinsically variable star-planet interaction [Shkolnik et al., 2008]. As of today, there is still no firm detection of such an interaction, in spite of the growing number of searches in the optical and $\mathrm{X}$-ray ranges [Poppenhaeger et al., 2011; and references therein]. HD 179949 and $v$ And nevertheless remain favoured targets for radio observations.

As explained above, the magnetospheres of hot jupiters could be strong radio emitters. But this implies that the planetary magnetic field is large enough so that cyclotron frequencies in the vicinity of the planet are $>10 \mathrm{MHz}$. But as hot jupiters are likely to be spin-orbit synchronized (synchronization time scales are of the order of $10^{5-6}$ years [Baraffe et al., 2010]), their sidereal rotation is relatively slow - a few days period instead of 10 hours for Jupiter -, and their magnetic dynamo and the resulting magnetic field may have decayed to low values. This question was addressed by Sanchez-Lavega [2004] and Reiners and Christensen [2010], who suggest that young massive fast-rotating planets should have a very strong magnetic field, and that the field could remain as high as several Gauss after synchronization and even for billion years-old planets. Radio detection will provide a crucial test of these theories.

Even if the exoplanet is unmagnetized or weakly magnetized, it may undergo a strong interaction with the magnetic field of its parent star. As the solar/stellar wind is transalfvénic at 5-10 stellar radii from the star [Zarka, 2007], the nature of this interaction is very similar to that between Io and Jupiter, so-called "unipolar inductor", with the planet playing the role of Io and the star playing that of Jupiter. This interaction was studied by Willes and $\mathrm{Wu}[2004,2005]$ for the case of a terrestrial planet orbiting a white dwarf (or for a binary white dwarf). It has been noticed that the star must be strongly magnetized $(\geq 30-100 \mathrm{G})$ so that the CMI can operate in its corona [Zarka et al., 2001; Zarka, 2007]. As a consequence, the radio emission induced by the planet in the stellar magnetic field may reach high frequencies (100's of $\mathrm{MHz}$ or $\geq 1 \mathrm{GHz}$ ). It was also shown that the unipolar inductor interaction follows the "radio-to-magnetic" scaling law, so that extrapolation to 
the case of hot jupiters orbiting strongly magnetized stars may lead to radio emissions up to $10^{6} \times$ that of Jupiter [Zarka, 2006, 2007]. Application of this scaling law to the energy budget of the star-planet interaction leading to the tentatively observed chromospheric spot in HD 179949 implies a stellar magnetic field of 30-100 G and/or a strong stellar wind and/or a large planetary magnetic field. Here again, radio observations will bring crucial informations.

In order to test the possible saturation of this scaling law toward large Poynting fluxes and high radio powers, Zarka [2010] compared to its predictions the detection at $8.4 \mathrm{GHz}$ of the RS CVn magnetic binary V711 $\tau$. The detected level $\sim 100$ mJy from a range of $35 \mathrm{pc}$ [Budding, 1998] implies an emitted radio power $\sim 7 \times 10^{19} \mathrm{~W}$, while the dissipated power - assuming a field strength $\sim 10$ Gauss, an inter-star stream velocity $\sim 300 \mathrm{~km} / \mathrm{s}$, and a characteristic size of interaction $\sim 10^{8} \mathrm{~m}-$ is about $\sim 7 \times 10^{21} \mathrm{~W}$. Although uncertainties on these estimates are large, the corresponding point falls remarkably close to the prediction of the "radio-to-magnetic" scaling law, suggesting that it may remain valid up to 10 orders of magnitude above the solar system planets range. Jardine and Cameron [2008] have proposed a theoretical basis for the "radio-to-magnetic" scaling law: invoking magnetic reconnection, production of parallel electric fields, and runaway electron acceleration at the exoplanet's magnetopause, these authors derived the slope $\sim 1$ of the scaling law, the proportionality constant remaining an empirically determined parameter.

Many other "predictions" papers have been published, bringing quantitative - but not conceptual - additions to the previous works: role of stellar age, influence of Coronal Mass Ejections, stellar wind modeling and estimates of its strength via the stellar X-ray luminosity, modelling of the topology of the star-planet magnetic interaction... There is no room for citing all these papers here, but most of them are discussed in [Chian et al., 2010] (together with the role of plasma turbulence on radio emission generation in exoplanetary magnetospheres). The above scaling laws have been applied to the exoplanet census by Lazio et al. [2004] (considering only the "radio-to-kinetic" scaling law) and by Griessmeier et al. [2007] (considering several scaling laws including the "radio-tomagnetic" one).

Recently, Nichols [2011] presented an original approach, extrapolating the physics of Jupiter's rotation dominated magnetosphere to the case of exoplanets. At Jupiter, most of the auroral emissions - including the radio emissions independent of the satellites - do not depend on the solar wind-planet interaction, but rather on internal processes. Those include a dominant internal plasma source (Io), ionosphere-magnetosphere coupling, and associated breakdown of the plasma corotation in the magnetosphere [Cowley and Bunce, 2001]. Nichols [2011] found that rapidly rotating giant planets irradiated by a strong stellar X-UV flux and orbiting beyond $\sim 1$ AU from their parent star should generate intense ionosphere-magnetosphere current systems, and associated radio emissions up to $10^{4-5} \times$ Jupiter's. This result expands the parameter space for searching exoplanetary radio emissions, by including "regular" jupiters (with orbital semi-major axis $\geq 1$ AU) around strong X-UV emitting stars. 


\section{Recent Low-frequency Observations}

We carried out a second large search program at UTR-2 in 2007-2011, based on new digital receivers developed for that purpose. These receivers provide high resolutions, both spectral $(4 \mathrm{kHz})$ and temporal $(<1 \mathrm{msec} / \mathrm{spectrum})$, plus the possibility to record the cross-product between the signals from the two branches of UTR-2 [Ryabov et al., 2010]. Their stability is much higher than that of the AOS, and the observations were performed during the last (and very long!) solar minimum, under a little perturbed ionosphere. Seven observation campaigns were performed between march 2007 and january 2011, representing nearly 90 nights (or $>700$ hours) on $\sim 20$ main and $\sim 25$ secondary exoplanetary targets, including most of the above cited targets as well as the hot jupiters for which a star-planet interaction is suspected [Shkolnik et al., 2008], and those orbiting a star whose magnetic field has been measured (see below).

All observations have been performed in dual-beam (On \& Off) mode, over the 12-32 $\mathrm{MHz}$ band. Each beam is the 30' pencil beam resulting from the product of UTR2's EW and NS beams, calculated directly or deduced from phase switching (i.e. as $\left.(\mathrm{EW}+\mathrm{NS})^{2}-(\mathrm{EW}-\mathrm{NS})^{2}\right)$. Confusion is limited by the relatively small size of this beam (at these low frequencies) and the fact that each source is tracked, searching for a time varying emission. On and Off beams were separated by $1^{\circ}$ in the North-South direction. The resulting $>12$ Tbytes of high resolution dynamic spectra are being analyzed. The main step is the new automated RFI mitigation software that has been developed to process the recorded data in which, as previously, the signal of interest - if present - is "hidden", in the raw high resolution data, in the sky background fluctuations. A typical example of RFI identification and suppression is given in Figure 2. Following steps of the analysis include calibration by the sky background and subtraction of this background to obtain a zero-mean signal, and parametric dedispersion followed by $\mathrm{t}-\mathrm{f}$ integration over unpolluted pixels following equations (7) and (8) of [Zarka et al., 1997]. Then, time variations will be searched in the reduced data at the orbital period of the corresponding exoplanet observed.
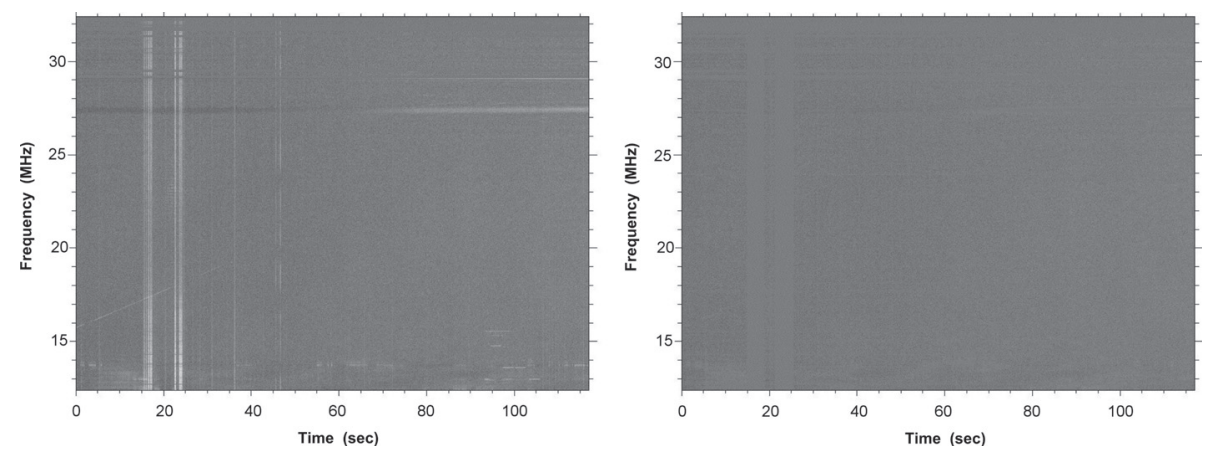

Figure 2: Left: Raw dynamic spectrum over $2 \mathrm{~min} . \times 20 \mathrm{MHz}$ (with total dynamic range $\sim 1 \mathrm{~dB}$ ). Right: Same after automatic RFI identification and suppression. 
Weak pulsars and Saturn's lightning (SED) were also observed as test sources. Figure 3 shows examples of SED and weak pulsar detection. The former illustrates the usefulness of dual beam observations, and the latter that of RFI mitigation. Both detections translate into a sensitivity of $\sim 1 \mathrm{Jy}$ at $1 \sec \times 1 \mathrm{MHz}$ integration.
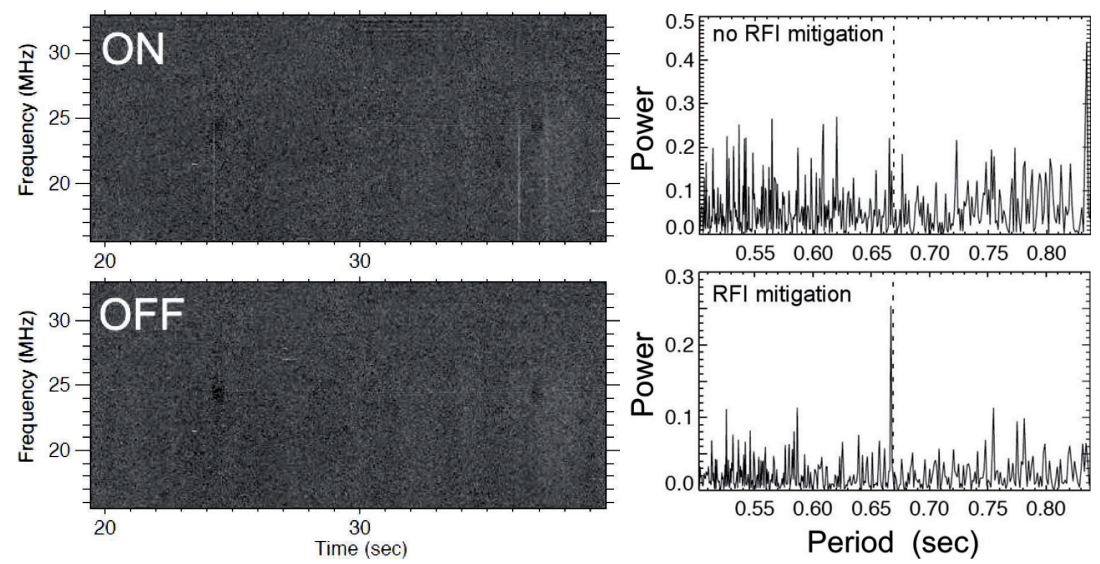

Figure 3: Left: Detection of broadband SED (near second 24, 36 \& 37) with UTR-2, in "On" dynamic spectrum only, at resolution of $20 \mathrm{msec} \times 4 \mathrm{kHz}$. Their typical flux density is $\sim 100 \mathrm{Jy}$. Right: Synchronous detection of the weak pulsar PSR 1322+83 (flux =36 mJy at $100 \mathrm{MHz}$, $D M=13.27$ pc.cm ${ }^{-3}, P=0.67 \mathrm{sec}$, pulse width $\left.\sim 0.03 \times P\right)$, after RFI mitigation only, in $500 \mathrm{sec}$ (15 sec effective) $\times 20 \mathrm{MHz}$ integration.

New radio observations were also performed by Lecavelier des Etangs et al. [2009] with the GMRT at 244 and $614 \mathrm{MHz}$. The target was the transiting exoplanet HD 189733 $\mathrm{B}$, for which spectropolarimetry has estimated a stellar magnetic field of $40 \mathrm{G}$. Images and time series encompassing the planet's eclipse behind the star provided no clue for a planetary radio signal. This may be due to a too high frequency of observation, selected for the very high sensitivity available ( $\sim 1 \mathrm{mJy}$ with a few minutes integration).

At such high frequencies, it is interesting to estimate if the incoherent synchrotron emission from extrasolar planets' radiation belts is detectable. Unfortunately, the level of Jupiter's synchrotron emission ( $\sim 5$ Jy at 4-5 AU range [de Pater, 2004]) corresponds to only $3 \times 10^{-11}$ Jy at 10 pc range. Even with an emission $10^{2} \times$ more intense than Jupiter's, the flux density at Earth will not exceed a few nJy, whereas the minimum detectable flux reachable at GMRT is of the order of a few $\mu \mathrm{Jy}$, even with long integrations.

\section{Method and Motivations}

Due to the limited angular resolution available at long wavelengths, it will remain impossible to resolve a star-exoplanet system. The search strategy consists thus in (1) detecting 
a signal, (2) determining if it comes from the star or the planet, and (3) studying its dynamic spectrum in intensity and polarization to derive new physical information on the observed system. Step (1) is the most difficult one, and still unsuccessful. Once detection will be achieved, star-planet discrimination (2) should be relatively easy using the emission polarization (highly circular/elliptical for all solar system planetary radio emissions but not for solar emissions) and its periodicity (orbital, especially). Beyond the validation of the above scaling laws that will be a direct consequence of detection, step (3) has been recently analyzed quantitatively by Hess and Zarka [2011], and is expected to provide through comparison of observations and modeling results:

- the identification of the star-planet interaction at work (magnetospheric with a radiosource fixed in longitude or in local time, or unipolar inductor);

- the measurement of the planetary magnetic field magnitude (estimated via the highest frequencies at which radio emission is detected), tilt and offset (estimated from the frequency ranges and relative phases of recurrence of radio emissions with opposite circular polarizations, originating from the two opposite magnetic hemispheres); these will put strong constraints on the planet's internal structure;

- the measurement of the planetary rotation period (via the periodic variation of the radio emissions), allowing to test directly spin-orbit synchronization;

- access to the inclination of the planetary orbit (that can be deduced from the highand low-frequency cutoffs of the emissions from opposite magnetic hemispheres.

More generally, radio detection of exoplanets, if better than marginal, will considerably expand the field of comparative magnetospheric physics to star-planet plasma interactions at large. The existence of exoplanetary magnetospheres also has implications for exobiology through limitation of the atmospheric erosion and cosmic ray bombardment [Griessmeier et al., 2004; Lammer et al., 2006].

\section{Perspectives}

Strong expectations for the radio detection and study of exoplanets are carried by the new giant european low-frequency array LOFAR, presently in commissionning phase [Girard et al., 2011]. LOFAR is a spectro-imager in the $30-250 \mathrm{MHz}$ range, with an effective area up to $2 \times 10^{5} \mathrm{~m}^{2}$, and a sensitivity down to the mJy level. It can produce 1 secintegrated images in its interferometric mode, as well as high resolution dynamic spectra in coherent phased-array mode (see e.g. [Stappers et al., 2011] and www.lofar.org). It has built-in multi-beam, RFI mitigation, and ionospheric calibration capabilities. Radio exoplanet search is the subject of a working group of LOFAR's "Transients" Key Scientific Project. First targeted observations are expected in 2011, as well as piggybacking on 1 sec imaging surveys. The LOFAR frequency range is very complementary to the UTR-2 one. Together, they allow us to address both magnetospheric and unipolar inductor emissions (Figure 4). 

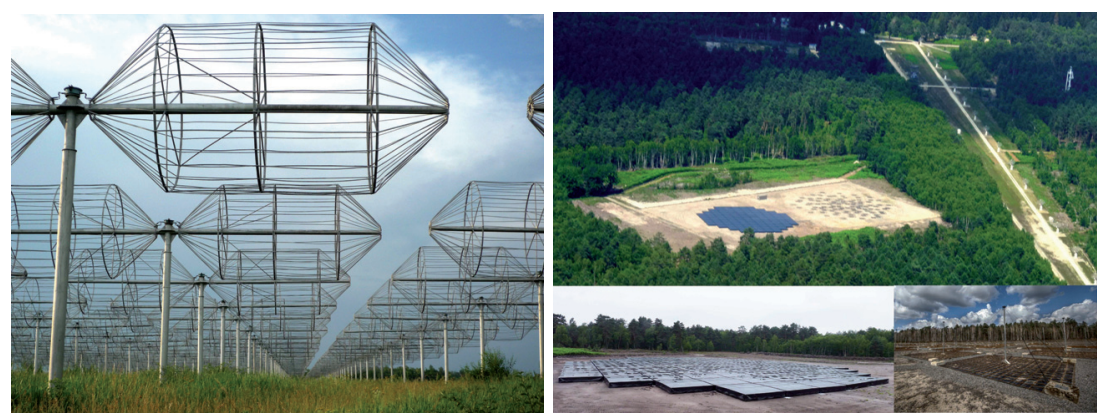

Figure 4: Left: UTR-2 antennas in Kharkov. Right: the LOFAR station in Nançay and below it a zoom on its HF and LF antennas (photos I. Thomas \& I. Cognard).

Target selection will rely in particular on recent results from optical spectropolarimetry (Stokes V Doppler imaging), that provide estimates of stellar magnetic field strength and topology. For example, minimum field strengths of a few Gauss have been measured for $\tau$ Boo and HD 179949, 40 G for HD 189733 [Farès, 2011], and up to 500 G for HD 171488. Reconstruction of the field topology up to the planet's orbit allowed Farès et al. [2010] to predict possible time variations of its magnetospheric radio emission. This will help selecting optimal orbital phases for future radio oservations. MHD modelling of magnetized stellar winds [Vidotto et al., 2010; and references therein] will bring additional guidance.

On the longer term, SKA will provide an effective area $\sim 1 \mathrm{~km}^{2}$ in the range $0.1-25 \mathrm{GHz}$, a sensitivity down to the $\mu \mathrm{Jy}$ level, and angular resolution down to a few milliarcseconds. It will permit to address unipolar inductor emission from star-planet systems (including hot earths around white dwarfs [Willes and Wu, 2004, 2005]), as well as radio astrometry (of planets around $\mathrm{M}$ dwarfs) or detection of artificial emissions. In order to expand the spectral range down to lower frequencies $(\leq 1 \mathrm{MHz})$ and thus access lower planetary and stellar field strengths, ESA and NASA projects of radio arrays on the radio-quiet Lunar far side are very appealing. Precursors submitted to ESA include SURO and the Farside Explorer.

Radio exoplanet search is a recent, still arid but highly motivating and potentially highly rewarding field, that wonderfully complements - scientifically and technically - planetary radio emission studies with high $\mathrm{S} / \mathrm{N}$ in our solar system.

Acknowledgements. The works reviewed here involved many collaborators: C. Barrow, B. Cecconi, A. Coffre, L. Denis, M.D. Desch, P. Dierich, C. Fabrice, H. Falcke, R. Farès, W.M. Farrell, A. Gerbault, J. Girard, J.-M. Griessmeier, S. Hess, A. Konovalenko, R. Kozhin, T.J. Lazio, W. Majid, C. Moutou, J. Nichols, J. Queinnec, P. Ravier, C. Rosolen, H.O. Rucker, B.P. Ryabov, V.B. Ryabov, V.A. Shevcenko, M. Sidorchuk, H. Spreeuw, R. Treumann, D. Vavriv, V. Vinogradov, R. Weber. D. Winterhalter, G. Woan, S. Zakharenko, et al. (apologies for those not mentioned here). I also acknowledge the support of the ANR program NT05-1_42530 "Radio-Exopla" (2006-2010). 


\section{References}

Baraffe, I., G. Chabrier, and T. Barman, The physical properties of extra-solar planets, Rep. Prog. Phys., 73, 016901, 2010.

Bastian, T.S., G. A. Dulk, and Y. Leblanc, A search for radio emission from extrasolar planets, Astrophys. J., 545, 1058-1063, 2000.

Braude, S. Ya., A. V Megn, B.P. Ryabov, N. K. Sharykin, and I. N. Zhouck, Decametric survey of discrete sources in the northern sky: I. the UTR-2 radiotelescope. Experimental techniques and data processing, Astrophys. Space Sci., 54, 1, 3-36, 1978.

Budding, E., O. B. Slee, and K. Jones, Further discussion of binary star radio survey data, Publ. Astron. Soc. Austr., 15, 183, 1998.

Burke, B. F., Prospects for the study of planetary radio emission, in Planetary Radio Emissions III, edited by H. O. Rucker, S. J. Bauer, and M. L. Kaiser, Austrian Academy of Sciences Press, Vienna, 485-488, 1992.

Chian, A. C.-L., M. Han, R.A. Miranda, C. Shu, and J.A. Valdivia, The planetaryexoplanetary environment: a nonlinear perspective, Adv. Space Res., 46, 472-484, 2010 .

Cowley, S.H., and E. J. Bunce, Origin of the main auroral oval in Jupiter's coupled magnetosphere-ionosphere system, Planet. Space Sci., 49, 1067-1088, 2001.

Cuntz, M., S. H. Saar, and Z. E. Muzeliak, On stellar activity enhancement due to interactions with extrasolar giant planets, Astrophys. J., 533, L151-L154, 2000.

de Pater, I., and P. Zarka, LOFAR and Jupiter's radio (synchrotron) emissions, Planet. Space Sci., 52, 1449-1454, 2004.

Desch, M. D., and M.L. Kaiser, Predictions for Uranus from a radiometric Bode's law, Nature, 310, 755, 1984.

Farès, R., Magnétism, activité et interactions magnétosphériques dans les systèmes étoile / planète géante proche (Jupiter chaud), Thèse de Doctorat, Univ. Toulouse, 2011.

Farès, R., J.-F. Donati, C. Moutou, M. M. Jardine, J.-M. Griessmeier, P. Zarka, E. Shkolnik, D. Bohlender, C. Catala, A. C. Cameron, and G. A. H. Walker, Searching for Star-Planet interactions within the magnetosphere of HD 189733, Mon. Not. Roy. Astron. Soc., 406, 409-419, 2010.

Farrell, W. M., M. D. Desch, and P. Zarka, On the possibility of coherent cyclotron emission from extrasolar planets, J. Geophys. Res., 104, 14025-14032, 1999.

Farrell, W. M., T. J. Lazio, M. D. Desch, T. Bastian, and P. Zarka, Limits on the Magnetosphere/Stellar Wind Interactions for the Extrasolar Planet about Tau Bootes, ASP Conf. Ser. 294: Scientific Frontiers in Research on Extrasolar Planets, 151-156, 2003 . 
Farrell, W. M., T. J. W. Lazio, M. D. Desch, T.S. Bastian, and P. Zarka, Radio Emission from Extrasolar Planets, Bioastronomy 2002: Life Among the Stars, IAU Symp. 213, edited by R. Norris, and F. Stootman, Astron. Soc. of the Pacific, San Francisco, 73, 2004a.

Farrell, W. M., T. J. W. Lazio, P. Zarka, T. Bastian, M. D. Desch, and B. P. Ryabov, The radio search for extrasolar planets with LOFAR, Planet. Space Sci., 52, 1469-1478, 2004 b.

Galopeau, P.H. M., M. Y. Boudjada, and H. O. Rucker, Evidence of jovian active longitude: 1. Efficiency of cyclotron maser instability, J. Geophys. Res., 109, A18, 12217, 2004.

George, S. J., and I. R. Stevens, Giant Metrewave Radio Telescope low-frequency observations of extrasolar planetary systems, Mon. Not. Roy. Astron. Soc., 382, 455-460, 2007.

George, S. J., and I. R. Stevens, A deep 150MHz Giant Metrewave Radio Telescope survey in Eridanus, Mon. Not. Roy. Astron. Soc., 390, 741-751, 2008.

Girard, J. N., Zarka, P., M. Tagger, L. Denis, D. Charrier, and A. Konovalenko, Antenna design and distribution for a LOFAR Super Station in Nançay, in Planetary Radio Emissions VII, edited by H. O. Rucker, W. S. Kurth, P. Louarn, and G. Fischer, Austrian Academy of Sciences Press, Vienna, this issue, 495-503, 2011.

Griessmeier, J.-M., A. Stadelmann, T. Penz, H. Lammer, F. Selsis, I. Ribas, E. F. Guinan, U. Motschmann, H. K. Biernat, and W. W. Weiss, The effect of tidal locking on the magnetospheric and atmospheric evolution of "hot Jupiters", Astron. Astrophys., 425, 753-762, 2004.

Griessmeier, J.-M., P. Zarka and H. Spreeuw, Predicting low-frequency radio fluxes of known extrasolar planets, Astron. Astrophys., 475, 359-368, 2007.

Hess, S. L. G., and P. Zarka, Modeling the radio signature of the orbital parameters, rotation, and magnetic field of exoplanets, Astron. Astrophys., 531, A29, doi:10.1051/ 0004-6361/201116510, 2011.

Hilgers, A., The auroral radiating plasma cavities, Geophys. Res. Lett., 19, 237, 1992.

Jardine, M., and A.C. Cameron, Radio emission from exoplanets: the role of the stellar coronal density and magnetic field strength, Astron. Astrophys., 490, 843-851, 2008 .

Lammer, H., M. L. Khodachenko, H. I. M. Lichtenegger, J.-M. Griessmeier, H. L. Biernat, and H. O. Rucker, Atmospheric erosion of weakly magnetized Hot Jupiter's, European Planetary Science Congress, Berlin, Germany, 388, 2006.

Lazio, T. J. W., and W. M. Farrell, Magnetospheric Emissions from the Planet Orbiting $\tau$ Bootis: A Multiepoch Search, Astrophys. J., 668, 1182-1188, 2007.

Lazio, T. J. W., W. M. Farrell, J. Dietrick, E. Greenlees, E. Hogan, C. Jones, and L. A. Hennig, The radiometric Bode's law and extrasolar planets, Astrophys. J., 612, 511-518, 2004 . 
Lazio, T. J. W., S. Carmichael, J. Clark, E. Elkins, P. Gudmundsen, Z. Mott, M. Szwajkowski, and L. A. Hennig, A Blind Search for Magnetospheric Emissions from Planetary Companions to Nearby Solar-Type Stars, Astron. J., 139, 96-101, 2010a.

Lazio, T. J. W., P. D. Shankland, W. M. Farrell, and D. L. Blank, Radio Observations of HD 80606 Near Planetary Periastron, Astron. J., 140, 1929-1933, 2010 b.

Le Quéau, D., Planetary radio emissions from high magnetic latitudes: the "CyclotronMaser" theory, in Planetary Radio Emissions II, edited by H. O. Rucker, S. J. Bauer, and B. M. Pedersen, Austrian Academy of Sciences Press, Vienna, 381-398, 1988.

Lecacheux, A., On the Feasibility of Extra-Solar Planetary Detection at Very Low Radio Frequencies, in Bioastronomy: The Search for Extraterrestial Life - The Exploration Broadens, edited by J. Heidmann and M. J. Klein, Lecture Notes in Physics, Berlin Springer Verlag, 390, 21-30, 1991.

Lecavelier des Etangs, A., S.K. Sirothia, Gopal-Krishna, and P. Zarka, GMRT radio observations of the transiting extrasolar planet HD189733 b at 244 and $614 \mathrm{MHz}$, Astron. Astrophys., 500, L51-L54, 2009.

Louarn, P., Radio emissions from filamentary sources: A simple approach, in Planetary Radio Emissions IV, edited by H. O. Rucker, S. J. Bauer, and A. Lecacheux, Austrian Academy of Sciences Press, Vienna, 153-165, 1997.

Majid, W., D. Winterhalter, I. Chandra, T. Kuiper, J. Lazio, C. Naudet, and P. Zarka, Search for radio emission from extrasolar planets: Preliminary analysis of GMRT data, in Planetary Radio Emissions VI, edited by H. O. Rucker, W. S. Kurth, and G. Mann, Austrian Academy of Sciences Press, Vienna, 589-594, 2006.

Manning, R., and G.A. Dulk, The galactic background radiation from 0.2 to $13.8 \mathrm{MHz}$, Astron. Astrophys., 372, 663-666, 2001.

Mayor, M. and D. Queloz, A Jupiter-mass companion to a solar-type star, Nature, 378, 355-359, 1995.

Nichols, J., Magnetosphere-ionosphere coupling at Jupiter-like exoplanets with internal plasma sources: implications for detectability of auroral radio emissions, Mon. Not. Roy. Astron. Soc., 469, 2011.

Poppenhaeger, K., L. F. Lenz, A. Reiners, J. H. M. M. Schmitt, and E. Shkolnik, A search for star-planet interactions in the $v$ Andromedae system at X-ray and optical wavelengths, Astron. Astrophys., 528, A58, 2011.

Preusse, S., A. Kopp, J. Buchner, and U. Motschmann, A magnetic communication scenario for hot Jupiters, Astron. Astrophys., 460, 317-322, 2006.

Raterron, J.-M., Réalisation d'un Spectrographe Acousto-Optique: Analyse et traitement de l'information, Thèse de Doctorat, Observatoire de Paris-Meudon/Université ParisSud (XI), Orsay, 1985.

Reiners, A., and U.R. Christensen, A magnetic field evolution scenario for brown dwarfs and giant planets, Astron. Astrophys., 522, A13, 2010. 
Ryabov, V. B., P. Zarka, and B. P. Ryabov, Search of exoplanetary radio signals in the presence of strong interference : Enhancing sensitivity by data accumulation, Planet. Space Sci., 52, 1479-1491, 2004.

Ryabov, V. B., D. M. Vavriv, P. Zarka, B. P. Ryabov, R. Kozhin, V. V. Vinogradov, and L. Denis, A low-noise, high dynamic range digital receiver for radio astronomy applications: an efficient solution for observing radio-bursts from Jupiter, the Sun, pulsars and other astrophysical plasmas below $30 \mathrm{MHz}$, Astron. Astrophys., 510, A16, 2010.

Saar, S.H., and M. Cuntz, A search for Ca II emission enhancement in stars resulting from nearby giant planets, Mon. Not. Roy. Astron. Soc., 325, 55-59, 2001.

Sanchez-Lavega, A., The magnetic field in giant extrasolar planets. Astrophys. J., 609, L87-L90, 2004.

Shkolnik, E., G. A. H. Walker, and D. A. Bohlender, Evidence for planet-induced chromospheric activity on HD 179949, Astrophys. J., 597, 1092-1096, 2003.

Shkolnik, E., G. A. H. Walker, D. A. Bohlender, P.-G. Gu, and M. Kurster, Hot Jupiters and hot spots: the short- and long-term chromospheric activity on stars with giant planets, Astrophys. J., 622, 1075-1090, 2005.

Shkolnik, E., D. A. Bohlender, G. A.H. Walker, and A. C. Cameron, The On/Off nature of star-planet interactions, Astrophys. J., 676, 628-638, 2008.

Stappers, B.W., et al. (93 co-authors), Observing Pulsars and Fast Transients with LOFAR, Astron. Astrophys., 530, A80, doi:10.1051/0004-6361/201116681, 2011.

Vidotto, A. A., M. Opher, V. Jatenco-Pereira, and T. I. Gombosi, Simulations of winds of weak-lined T Tauri stars. II. The effects of a tilted magnetosphere and planetary interactions, Astrophys. J., 720, 1262-1280, 2010.

Willes, A. J., and K. Wu, Electron-cyclotron maser emission from white dwarf pairs and white dwarf planetary systems, Mon. Not. Roy. Astron. Soc., 348, 285-296, 2004.

Willes, A.J., and K. Wu, Radio emissions from terrestrial planets around white dwarfs, Astron. Astrophys., 432, 1091-1100, 2005.

Winglee, R. M., G.A. Dulk, and T.S. Bastian, A search for cyclotron maser radiation from substellar and planet-like companions of nearby stars, Astrophys. J. Lett., 309, L59-L62, 1986.

Winterhalter, D., T. Kuiper, W. Majid, I. Chandra, J. Lazio, P. Zarka, C. Naudet, G. Bryden, W. Gonzalez, and R. Treumann, Search for radio emissions from extrasolar planets : the observation campaign, in Planetary Radio Emissions VI, edited by H. O. Rucker, W.S. Kurth, and G. Mann, Austrian Academy of Sciences Press, Vienna, 595-602, 2006.

Wolszczan, A., and D. A. Frail, A planetary system around the millisecond pulsar PSR1257 + 12, Nature, 355, 145-147, 1992. 
Yantis, W.F., W.T. Sullivan, III, and W. C. Erickson, A Search for Extra-Solar Jovian Planets by Radio Techniques, Bull. Am. Ast. Soc., 9, 453, 1977.

Zarka, P., D. Le Quéau, and F. Genova, The Maser Synchrotron Instability in an inhomogeneous medium: Determination of the spectral intensity of Auroral Kilometric Radiation, J. Geophys. Res., 91, 13542-13558, 1986.

Zarka, P., The auroral radio emissions from planetary magnetospheres: what do we know, what don't we know, what do we learn from them? Adv. Space Res., 12, 8, 99, 1992.

Zarka, P., Recherche de planètes extra-solaires sur ondes décamétriques, in La détection au sol de planètes extrasolaires, CNRS/Observatoire de Haute-Provence/Institut Gassendi (Marseille), 1995.

Zarka, P., Auroral Radio Emissions at the Outer Planets: Observations and Theories, J. Geophys. Res., 103, 20159-20194, 1998.

Zarka, P., Hot Jupiters and magnetized stars: Giant analogs of the satellite-Jupiter system?, in Planetary Radio Emissions VI, edited by H. O. Rucker, W.S. Kurth, and G. Mann, Austrian Academy of Sciences Press, Vienna, 543-570, 2006.

Zarka, P., Plasma interactions of exoplanets with their parent star and associated radio emissions, Planet. Space Sci., 55, 598-617, 2007.

Zarka, P., Radioastronomy and the study of exoplanets, in Pathways Towards Habitable Planets, ASP Conf. Series, 430, edited by V. Coudé du Foresto, D. M. Gelino and I. Ribas, 175-180, 2010.

Zarka, P., B.P. Ryabov, V.B. Ryabov, R. Prange, M. Abada-Simon, T. Farges, and L. Denis, On the Origin of Jovian Decameter Radio Bursts, in Planetary Radio Emissions IV, edited by H. O. Rucker, S. J. Bauer, and A. Lecacheux, Austrian Academy of Sciences Press, Vienna, 51-63, 1997.

Zarka, P., R. A. Treumann, B. P. Ryabov, and V. B. Ryabov, Magnetically-driven planetary radio emissions and application to extrasolar planets, Astrophys. Space Sci., 277, 293-300, 2001.

Zarka, P., B. Cecconi, and W.S. Kurth, Jupiter's low-frequency radio spectrum from Cassini/Radio and Plasma Wave (RPWS) absolute flux density measurements, J. Geophys. Res., 109, A09S15, 2004. 
